\title{
ACTION PUBLIQUE ET PARTIS POLITIQUES
}

\section{L'analyse de l'agenda législatif français entre 1981 et $2009{ }^{1}$}

\author{
Simon Persico ${ }^{2}$, Caterina Froio ${ }^{3}$ \\ et Isabelle Guinaudeau ${ }^{4}$
}

Résumé : La compétition entre projets partisans est souvent citée comme un élément constitutif de toute démocratie représentative. Si les littératures francophone et anglosaxonne ont appréhendé différemment le lien entre compétition partisane et politiques publiques, les partis sont partout le parent pauvre des théories de l'action publique. S'inspirant des théories de la mise sur agenda et de la compétition sur enjeux, cet article plaide pour une reconsidération du poids de la variable partisane, en adoptant une approche à la fois globale et différenciée visant à établir dans quelles conditions les partis aux affaires influencent les politiques publiques. Pour cela, les données du Comparative Agendas Project offrent un instrument privilégié, dont nous illustrons les potentialités en testant plusieurs hypothèses sur le cas des activités législatives françaises entre 1981 et 2009.

MOTS-CLÉS : MISE SUR AGENDA - FRANCE - COMPÉTITION SUR ENJEUX - PARTIS POLITIQUES - PRODUCTION LÉGISLATIVE

\begin{abstract}
Competition among partisan projects is often cited as a constitutive element of all representative democracies. While the Francophone and English-medium research literature have viewed the link between partisan competition and public policy differently, in both cases parties tend to be the "poor cousins" of theories of public action. Drawing from theories of agenda-setting and issue competition, this article argues for taking the partisan variable more seriously, by adopting an approach which is both global and differentiated and which aims to establish in what conditions governing parties influence public policies. Data from the Comparative Agendas Project provide a challenging basis for investigating this issue. Testing several hypotheses concerning French legislative activities between 1981 and 2009 highlights its potentialities.
\end{abstract}

KEYWORDS: AGENDA-SETTING - FRANCE - ISSUE COMPETITION - POLITICAL PARTIES - LEGISLATIVE PRODUCTION

"To talk, today, about democracy, is to talk about a system of competitive parties. Unless one chooses to reject the representative model that has been the staple of the theory and practice of democracy since the French Revolution, one must come to terms with political parties " (Robertson, 1976, p. 1).

Comme l'illustre cette citation de David Robertson, la compétition entre organisations et projets partisans est communément citée comme un élément constitutif de toute démocratie

\footnotetext{
1. Cet article a pris forme dans le cadre du projet Agendas France, soutenu par l'ANR depuis 2009. Nous remercions vivement les responsables du projet, Sylvain Brouard et Emiliano Grossman, de nous avoir impliqués dans le codage des programmes électoraux et d'avoir mis à notre disposition les bases de données relatives aux lois promulguées.

2. Centre d'études européennes, Sciences Po.

3. Institut universitaire européen, Florence.

4. Max Weber Fellow à l'Institut universitaire européen, Florence.
} 
représentative ${ }^{5}$. Dans presque toutes les conceptions, les partis sont placés au cœur du processus démocratique, eu égard à leurs fonctions de sélection du personnel politique, d'élaboration de propositions programmatiques et de mise en œuvre de ces dernières une fois au pouvoir (Klingemann et al., 1994, p. 5 ; Manin, 1995). Pour le citoyen, voter pour un parti plutôt qu'un autre, c'est notamment faire un choix entre différentes options de politiques publiques (Aardal, van Wijnen, 2005). Pour les partis, les politiques publiques sont des "biens proposés " aux électeurs, parfois conçus comme des consommateurs, grâce auxquels ils se différencient sur le marché politique, (Gaxie, 2003, p. 24 ; voir aussi Downs, 1957). Les théories normatives de la démocratie se construisent donc à partir du postulat d'un lien fort entre politics et policies.

Or, les théories dominantes de l'action publique tendent soit à négliger l'étude de la variable partisane, soit à conclure à sa marginalité face à d'autres paramètres tels que les contraintes économiques, la friction institutionnelle ou encore l'inertie des choix antérieurs. Cet article part de la conviction que l'empreinte partisane sur les politiques publiques n'a pas été étudiée de façon assez systématique pour pouvoir évincer définitivement les partis des modèles du policymaking. Après avoir proposé un tour d'horizon des résultats disponibles sur le sujet, nous argumentons en faveur d'une démarche différenciée, visant à établir dans quelles conditions le ou les partis aux affaires influencent les politiques publiques. Les données collectées par le Comparative Agendas Project (CAP), qui permettent de comparer les agendas - notamment parlementaires, gouvernementaux, médiatiques et partisans - de nombreux pays, offrent un instrument privilégié pour appréhender cette problématique. Nous illustrons ces potentialités en testant plusieurs hypothèses sur le cas des activités législatives françaises entre 1981 et 2009 .

\section{La variable partisane, " second souffle de l'analyse des politiques publiques "?}

La question du lien entre composition partisane du gouvernement et changement des politiques publiques n'a pas suscité d'intérêt particulier dans le cadre de la science politique française. Comme l'expliquent Patrick Hassenteufel et Andy Smith (2002, p. 63), cet angle mort est sans doute lié à la partition disciplinaire de la science politique, notamment entre étude de l'action publique, d'une part, et analyse des partis et des élections, d'autre part. Les travaux francophones existants font le lien entre les deux perspectives à partir d'études de cas centrées sur une mesure ou un secteur de politique publique, en intégrant la dimension partisane. Leurs résultats mettent au jour une image partielle et contrastée. Certaines études soulignent les conséquences décisives de l'alternance dans certains domaines, à l'image de la politique du prix unique du livre, rendue possible par l'alternance de 1981 (Surel, 1997), de la réorientation de la politique du logement à partir de 1995, fruit des échanges entre le RPR de Jacques Chirac et de certains acteurs professionnels et associatifs (Zittoun, 2001), de la réforme du Code pénal de 1992 et de l'adoption du PACS en 1999 (Lascoumes, 2009). De même, la thèse d'A. Évrard (2010) démontre l'impact décisif des Grünen allemands sur les politiques énergétiques de leur pays. Au contraire, c'est l'absence de différence entre les deux grands partis de gouvernement en matière d'énergie qui explique la grande stabilité du programme nucléaire français depuis les années 1970 (Brouard, Guinaudeau, 2011 ; voir aussi Barthe, 2006). Ce type d'étude de cas offre un ensemble de résultats

5. Voir par exemple l'ouvrage classique de J. Schumpeter (1942). 
intéressants qui peuvent être mis en perspective, mais les tentatives de mesurer précisément l'influence des partis sur les politiques publiques dans leur ensemble restent rares (Coman, Persico, 2010) $)^{6}$.

Dans le monde anglo-saxon, au contraire, ce questionnement nourrit un ensemble de travaux depuis plusieurs décennies, dont la plupart se concentrent sur l'hypothèse de base selon laquelle les partis de gauche mèneraient des politiques plus keynesiennes que les partis de droite. L'attention s'est donc focalisée sur les dépenses publiques, présumées supérieures sous les gouvernements de gauche, et parfois sur leur allocation, les partis de gauche étant supposés privilégier les politiques sociales et éducatives, tandis que les partis de droite feraient la part belle aux politiques de défense et de sécurité. Peut-être du fait de cette focalisation sur les indicateurs macro-économiques, les conclusions sont là encore mitigées, voire franchement divergentes.

\section{La thèse de la neutralité partisane des politiques publiques}

Le postulat de l'influence partisane a été mis en cause dès les années 1960 par l'étude de P. Cutright sur les facteurs explicatifs du programme de National Security américain (1965). Ses conclusions sont partagées par G. Haniff (1976), qui s'est intéressé aux politiques sociales, ou encore par H. Wilenski (1975), qui a montré que la conjoncture économique et la situation démographique expliquent mieux l'évolution des dépenses sociales que la couleur politique du gouvernement. Les recherches plus récentes ont confirmé l'existence d'une série de facteurs faisant obstacle au changement des politiques, y compris en période d'alternance - mécanismes d'héritage (Rose, Davies, 1994 ; Pierson, 2000 ; Adler, Wilkerson, 2008), contraintes économiques liées à la mondialisation (Garrett, Lange, 1989 ; Keohane, Milner, 1996) et à l'intégration européenne (Guinaudeau, 2011) ou encore vieillissement des populations (Green-Pedersen, Wilkerson, 2006).

La thèse de la neutralité partisane va dans le sens de plusieurs observations importantes de l'étude des partis politiques et de l'action publique. D'une part, elle peut être lue en lien avec le passage du parti de masse au parti attrape-tout (Kirchheimer, 1966), puis au parti cartel (Katz, Mair, 1995), qui mènerait les partis à revoir leur stratégie de conquête du pouvoir en s'attachant à séduire l'ensemble des électeurs, quitte à s'éloigner de leur électorat traditionnel (Crouch, 2004 ; Kitschelt, 1999, pour le cas des partis sociaux-démocrates). Cette stratégie se traduirait en effet par une atténuation des conflits idéologiques et des préférences de politiques publiques qui y étaient classiquement associées (Mair, 2008).

D'autre part, l'idée d'une neutralité partisane corrobore l'acquis commun aux études de l'action publique selon lequel les élus jouent un rôle mineur dans le processus de décision. Sans dresser ici une liste exhaustive des courants qui traversent la discipline, on peut noter la concordance de vues qui règne pour constater l'innocuité des partis politiques, qu'il s'agisse de l'incrémentalisme de C. Lindblom (1959), qui décrit un processus de changement politique lent, impliquant des experts surmontant tant bien que mal les problèmes auxquels ils sont confrontés, de l'approche par les « réseaux de politiques publiques », formés par des bureaucrates et des experts souvent étrangers aux grandes orientations idéologiques portées par les partis (Heclo, 1974 ; Le Galès et al., 1995) ou encore des trois néo-institutionnalismes qui, s'ils diffèrent en de nombreux points (Hall, Taylor, 1996), s'accordent sur le fait que les responsables gouvernementaux n'ont qu'une capacité limitée à impulser le changement (PPC, 2010, p. 24). De même, la plupart des

6. On citera les travaux de F. Baumgartner et al. (2009), puis de C. Froio (2010) sur les agendas nationaux. 
approches cognitivistes s'accordent à reconnaître les limites de l'empreinte des partis au gouvernement. C'est notamment le cas de l'approche par les référentiels, référentiels jugés façonner les politiques publiques au-delà de l'inclinaison partisane du gouvernement (Jobert, 1994 pour l'exemple du référentiel néolibéral).

\section{La thèse de la différentiation partisane}

Ces conclusions sont pourtant contestées par de nombreux auteurs s'inscrivant dans une démarche similaire, focalisée sur les évolutions macro-économiques comme indicateur du changement. Sur la base de comparaisons à large échelle, les uns montrent que le niveau des dépenses publiques s'élève lorsque la gauche exerce le pouvoir (Cameron, 1978 ; Swank 1992), même si cet effet est modulé par le degré d'ouverture de l'économie (Hibbs, 1977, 1987). A. Blais, D. Blake et $\mathrm{S}$. Dion ont des résultats semblables, dans le cas de gouvernements mono-partisans (1993) comme de gouvernements de coalition (1996).

Les autres défendent l'hypothèse de la différentiation partisane en s'intéressant non plus au niveau général des dépenses publiques, mais à l'allocation différenciée des ressources budgétaires entre différents secteurs de politiques publiques. Ainsi, conformément à ce que leur idéologie et leur base électorale pourraient le donner à penser, les gouvernements de droite seraient plus enclins à soutenir les secteurs agricoles (Davis, Dempster, Wildavsky, 1966) et la défense (Boix, 2000 ; Brown, Cote, Lynn-Jones, 2000). Les politiques macroéconomiques (Boix, 2000 ; Wildavsky, 1987) et les politiques redistributives (Castles, 1982) des gouvernements de gauche diffèreraient aussi significativement de celles des gouvernements de droite, en particulier en période de crise.

D'autres travaux confirment aussi l'hypothèse de la différentiation partisane, en concentrant leur attention sur des domaines de politiques publiques particuliers. En matière d'immigration, par exemple, T. Bale et ses collègues offrent une analyse fine des changements impliqués par l'alternance gouvernementale au Royaume-Uni (Bale et al., 2011). Dans le cas de l'État-providence, W. Korpi et J. Palme montrent que, même en tenant compte d'autres facteurs, comme les points de veto constitutionnels, la situation économique ou les contraintes liées à la mondialisation, l'absence des partis sociaux-démocrates au pouvoir demeure largement explicative de la réduction des dépenses de l'État. Ces conclusions quantitatives sont confirmées dans des études de cas riches et nombreuses, comme celle de J. Levy (2001) sur les réformes économiques mises en œuvre sous le gouvernement Jospin, ou de S. Haüsermann (2010) sur le rôle des coalitions de partis et de syndicats dans la transformation des systèmes de retraite continentaux.

\section{Vers un renouvellement de l'analyse de l'action publique}

Si de nombreux travaux détaillent avec finesse les processus politiques, et souvent partisans, à l'œuvre dans l'implémentation d'une politique précise, force est de constater que les travaux cherchant à étudier le lien statistique entre parti au gouvernement et politiques mises en œuvre brossent un tableau mitigé, voire contradictoire. Ils tendent à donner raison aux "optimistes modestes qui pensent que cela change quelque chose qu'il y ait des élections ou non dans un pays » (Blais, 2003, p. 940), tout en relativisant la portée de la variable partisane ${ }^{7}$. Ces résultats

7. Plusieurs articles ont déjà fait une évaluation de ces études aux résultats divergents. Une méta-analyse portant sur 43 articles conclut qu' « environ $8 \%$ des évolutions des dépenses publiques s'explique par la variation dans la composition (gauche-droite) du gouvernement » (Imbeau, Pétri, Lamari, 2001, p. 23). Dans une étude statistique des résultats de 56 articles, P. Burstein et A. Linton (2002) estiment que l'hypothèse de la différentiation partisane n'est 
limités vont de pair avec des faiblesses communes aux travaux généralistes que nous venons de citer, qui ont souvent été pointées.

En premier lieu, en se concentrant exclusivement sur les budgets, ces travaux ne captent que le «produit fini » du policy process, un produit caractérisé par une forte stabilité (Ryu, 2009). Eu égard à l'inertie considérable des dépenses budgétaires de l'État (Siné, 2006 ; Bezes, Siné, 2011), le choix de limiter la variable dépendante censée indiquer le changement des politiques publiques au montant ou à la distribution des dépenses publiques n'est pas forcément le plus pertinent (von Beyme, 1985).

En outre, l'opérationnalisation de la principale variable indépendante - la variable partisane est souvent simpliste. Elle se limite généralement à une distinction binaire des partis entre gauche et droite, le parti de gauche étant supposé dépenser plus que le parti de droite. Cette opérationnalisation est donc aveugle aux préférences substantielles des partis. Pourtant, ce sont bien ces préférences qui devraient influencer les politiques publiques une fois le parti au pouvoir. Raisonner simplement en termes de gauche et de droite empêche ainsi de saisir la complexité des préférences d'un parti sur une multitude d'enjeux (Budge, McDonald, 2006), mais aussi de prendre en compte l'évolution de ces préférences. En 1997, par exemple, les dirigeants du Parti socialiste français promettaient non seulement de refuser « la privatisation des services publics et leur transformation en objet de profit » (Parti Socialiste, 1997, p. 13), mais aussi - entre autres - de créer un contrat unissant les couples de même sexe. Dans ces conditions, il semble discutable de nier l'impact de la coloration socialiste du gouvernement sur les politiques mises en œuvre entre 1997 et 2002, en constatant que le gouvernement Jospin est celui qui a le plus privatisé dans la période (Cos, 2011), sans prendre en considération d'autres actions, telles que la création du PACS.

L'opérationnalisation binaire de la variable partisane bute par ailleurs sur le défi de restituer l'évolution des préférences des partis. Les transformations du discours du parti travailliste britannique illustrent ce point avec éloquence. Les promesses du Labour de James Callaghan, Premier ministre britannique entre 1976 et 1979, ont très peu en commun avec celles du parti néotravailliste de Tony Blair, Premier ministre entre 1997 et 2007 et artisan principal d'un processus de «modernisation " du parti qui s'est traduit par la remise en cause profonde de ses analyses et principes keynesiens. Si l'on tient compte de ces changements, la priorité accordée par le gouvernement Blair à l'investissement privé (Faucher-King, Le Galès, 2007) ne peut pas être présentée comme l'expression d'une déconnexion entre programme du New Labour et politiques du gouvernement de Tony Blair.

II semble donc que les relations qui se nouent entre partis et politiques publiques puissent être mieux comprises en s'attachant, d'une part, à restituer plus précisément les préférences partisanes et en se concentrant, d'autre part, sur l'étape du policy process lors de laquelle l'hypothèse de l'effet partisan est la plus plausible : la mise sur agenda. Appréhendé plus précisément, l'impact de la variable partisane pourrait même apporter un "second souffle " à l'analyse des politiques publiques (Hassenteufel, Smith, 2002). Dans cette perspective, les théories de la mise sur agenda et de la compétition sur enjeux nous semblent offrir des outils d'analyse particulièrement prometteurs.

confirmée que dans $50 \%$ des cas environ. A. Blais, dans une autre méta-analyse, indique lui aussi que, toutes choses égales par ailleurs, un gouvernement de gauche dépense un peu plus qu'un gouvernement de droite (Blais, 2003, p. 940). 


\section{Affiner l'étude de la différentiation partisane en mobilisant les théories de la mise sur agenda et de la compétition sur enjeux}

\section{Mise sur agenda}

Le lien entre compétition partisane et politiques publiques peut être appréhendé de façon heuristique comme la connexion entre un agenda partisan et un agenda parlementaire. Au croisement des politiques publiques, de la sociologie politique, voire même des media studies (Hassenteufel, 2010) ${ }^{8}$, les théories de la mise sur agenda se prêtent bien à l'analyse de ce lien en restituant "l'ensemble des processus qui conduisent des faits sociaux à acquérir un statut de "problème public" ne relevant pas de la fatalité ou de la sphère privée et faisant l'objet de débats et de controverses médiatiques et politiques » (Garraud, 2010, p. 245). Dans son étude de la prise de décision en matière de fusions-acquisitions et de rémunération des PDG des grandes entreprises en France, en Allemagne, aux Pays-Bas et au Japon, P. Culpepper (2011) montre que la réactivité des législateurs vis-à-vis des préférences des électeurs est principalement déterminée par la visibilité des enjeux politiques en question. Les enjeux saillants et visibles dans les médias donneraient lieu à des décisions marquées par le souci de se conformer aux préférences des électeurs, tandis que les choix opérés dans les domaines plus discrets (que Culpepper appelle Quiet Politics) seraient plutôt le résultat de l'agrégation d'intérêts corporatistes. Cette étude comparative indique que la mise sur agenda d'un problème constitue une étape cruciale du changement politique.

L'étude de cette étape nous semble d'autant plus essentielle que les partis, de par leur accès privilégié aux arènes institutionnelles et médiatiques, disposent de ressources particulièrement développées pour imposer à l'agenda du champ politique les thèmes qui leur sont chers (Kleinnijenhuis, Rietberg, 1995). Si d'autres acteurs - experts, fonctionnaires, groupes d'intérêt - sont susceptibles de prendre le dessus lors des étapes subséquentes du policy-making, les partis ont probablement le plus grand rôle à jouer lors de cette phase de cristallisation des problèmes. De nombreuses études accordent d'ailleurs un poids considérable à la variable partisane dans la mise sur agenda politique.

C'est en particulier le cas de E. E. Schattschneider, dont beaucoup considèrent qu'il est le fondateur des approches en termes d'agenda-setting (Baumgartner, 2004, p. 288). Pour cet auteur, la constitution de partis politiques est l'une des étapes de l'expansion des conflits à des arènes politiques plus larges que la sphère privée. Cette expansion est donc surtout une bataille pour le contrôle de l'agenda politique (1960). L'ouvrage de référence de J. Kingdon (1984) considère lui aussi le rôle des partis politiques : l'agenda politique peut être affecté par «le contenu de leur plateforme, l'impact de leur leadership au congrès et, plus généralement, l'influence qu'ils peuvent avoir sur leurs sympathisants et l'idéologie qu'ils représentent » (p. 63-64). Pour J. Kingdon, les partis politiques sont au cœur du political stream dont la réunion avec le problem stream et le policy stream conditionne l'ouverture d'une fenêtre d'opportunité. F. Baumgartner et B. Jones $(2002,2005)$ incluent également les partis dans leur théorie de la mise sur agenda, même si leurs observations d'une grande stabilité de l'agenda du gouvernement et des législateurs laissent, somme toute, peu de place au politique : les changements, brutaux et durables, seraient plus liés à la friction institutionnelle qu'à l'alternance politique.

8. Nous nous concentrons ici sur l'agenda institutionnel, défini par R. Cobb et C. Elder comme « l'ensemble des objets considérés de manière active et sérieuse par l'organe institutionnel chargé de prendre les décisions » (1971, p. 906, voir aussi Cobb, Elder, 1972), dans la mesure où nous nous intéressons aux questions qui entrent in fine à l'ordre du jour du gouvernement et/ou du parlement. 
L'œuvre qui théorise le mieux le lien entre partis politiques et agenda institutionnel est sans doute celle de W. Riker. Ses travaux ont mis en lumière l'importance de deux phénomènes parallèles fondamentaux dans la compétition pour le contrôle de l'agenda : d'une part, la capacité à reformuler les termes du débat pour les tourner à son avantage - ce que l'auteur qualifie de rhetorics et qui sera repris par la suite dans une multitude de travaux portant sur le cadrage et la définition des problèmes (Riker, 1986 ; Riker, Calvert, Mueller, Wilson, 1996 ; voir aussi notamment : Carmines, Stimson, 1989 ; Rochefort, Cobb, 1994 ; Baumgartner, De Boef, Boydstun, 2008 ; Boussaguet, 2008). D'autre part, Riker met en avant la capacité à imposer le nombre et le type d'enjeux de politiques publiques sur l'agenda - ce que l'auteur qualifie d'heresthetics (Riker, 1993). C'est sous ce deuxième angle que notre article envisage d'étudier l'impact des partis politiques sur l'agenda institutionnel. En cela, nous faisons le lien entre les théories de la mise sur agenda - le versant politiques publiques - et des théories de la compétition sur enjeu le versant sociologie politique.

\section{Compétition sur enjeux}

La notion de compétition sur enjeu, inspirée par D. Stokes (1963), D. Robertson (1976), I. Budge et al. (1987) et W. Riker (1986), implique que chaque parti " possède » un ou plusieurs enjeux qui correspondent à son profil idéologique et au sujet desquels il est considéré comme compétent par les électeurs ${ }^{9}$. Les politiques environnementales, traditionnellement l'apanage des partis écologistes (Meguid, 2008) et l'État-providence et les politiques sociales, sujet de prédilection des partis sociaux-démocrates (Green-Pedersen, Blomqvist, 2004), en sont des exemples particulièrement parlants. Dans cette perspective, la compétition partisane prendrait la forme d'une lutte entre partis pour imposer les enjeux qui les avantagent sur l'agenda politique, tout en accordant le moins d'attention possible aux enjeux de leurs adversaires (Budge, Klingemann, Volkens et al., 2001 ; Klingemann, Volkens, Bara, 2006 ; Green-Pedersen, Mortensen, 2007). Partant, le parti vainqueur est censé privilégier ces mêmes enjeux dans les politiques mises en œuvre une fois arrivé au pouvoir (Budge, Hofferbert, 1990).

La théorie de la compétition sur enjeu s'intéresse finalement peu à la position que les partis adoptent sur chaque enjeu. Plus qu'un débat mettant en opposition des arguments contradictoires, la compétition partisane serait plutôt une lutte pour l'attention des médias et des électeurs. Si cette vision de la compétition électorale n'est pas exhaustive, elle permet d'expliquer certaines dynamiques des campagnes électorales. La capacité qu'a eue l'UMP d'imposer les thèmes de l'insécurité et de l'immigration dans la campagne électorale de 2007 a par exemple été analysée comme l'une des raisons principales de sa victoire à l'élection présidentielle, puis aux élections législatives en 2007 (Martin, 2010).

Le croisement des théories de la mise sur agenda et de la compétition sur enjeu permet ainsi d'appréhender la question du lien entre partis et politiques publiques à l'aune d'une problématique précise : les partis qui remportent les élections concentrent-ils leurs actions gouvernementales sur les domaines mis en avant lors de la campagne? Autrement dit, l'agenda parlementaire, issu du vote d'une majorité de députés représentant un parti ou une coalition de partis, reflète-t-il l'agenda électoral de ce(s) parti(s) $?^{10}$. Nous proposons ici une contribution à cette réflexion, élaborée à partir de cinq hypothèses de travail.

9. Sur la notion d'issue ownership, on se réfèrera à J. Petrocik (1996), ainsi qu'à É. Bélanger et B. Meguid (2008).

10. Plus précisément, nous nous intéressons ici à l'impact des thèmes mis en avant par le parti du Premier ministre sur les lois votées à l'Assemblée nationale pendant la législature. Si les débats sur la tendance au bipartisme en France sont loin d'être clos (Grunberg, Haegel 2007 ; Grossman, 2009), force est de constater que le parti du 


\section{Hypothèses}

Si la littérature classique développée autour du "Do parties matter ? " tend à conclure que la couleur politique du gouvernement a un effet significatif sur les dépenses publiques, il paraît plausible d'étendre l'argument à l'agenda parlementaire. Cela revient à considérer qu'un parti qui prend le pouvoir en mettant en avant certains enjeux devrait être incité à agir au sujet de ces mêmes enjeux, une fois aux affaires. Ainsi, notre première hypothèse est la suivante :

H1: Les thèmes politisés par le parti du Premier ministre lors de la précédente campagne électorale tendent à être érigés en priorités sur l'agenda parlementaire.

Au regard des conclusions contrastées des études existantes, il nous semble plus approprié de ne pas poser la question du "Do parties matter? " dans des termes absolus, mais de renouveler la réflexion en s'interrogeant sur les conditions qui affectent l'influence partisane, autrement dit sur le «When parties matter? ». La capacité d'une majorité à mettre en œuvre les politiques pour lesquelles elle a été élue varie probablement en fonction des institutions dans lesquelles son action s'inscrit. Ces institutions peuvent être caractérisées par le nombre de veto players susceptibles d'intervenir dans la production législative (Tsebelis, 2002), et donc possiblement de limiter l'impact du parti au gouvernement sur l'agenda politique. En France, deux institutions sont généralement considérées comme pouvant considérablement ralentir la procédure législative, même si l'on ne peut pas les considérer comme de véritables veto players au sens de G. Tsebelis (Brouard, 2009) : le Sénat, si sa majorité est différente de celle de l'Assemblée nationale (Conley, Bekafigo, 2006) ; et le président de la République, en cas de cohabitation (Lazardeux, 2008). Ces deux types de situations sont susceptibles de se traduire par une portée plus limitée de la variable partisane, ce qui permet d'élaborer deux hypothèses supplémentaires.

H2a : L'influence des préférences du parti du Premier ministre sur l'agenda législatif est moins forte en période de cohabitation.

H2b : L'influence des préférences du parti du Premier ministre sur l'agenda législatif est moins forte lorsque la majorité du Sénat diffère de celle de l'Assemblée nationale ${ }^{11}$.

Enfin, les travaux relatifs au comportement des acteurs partisans ont souligné que si la mise en œuvre des politiques publiques est un objectif des acteurs partisans, ces derniers peuvent aussi se fixer d'autres buts, en particulier la maximisation des votes et des sièges (Strom, Müller, 1999). Les arbitrages effectués entre ces trois objectifs sont susceptibles de conditionner l'influence partisane sur les politiques publiques en général et sur l'agenda parlementaire en particulier. Or, les études menées sur les effets des cycles électoraux montrent que les partis ont intérêt à mettre en œuvre leur mandat de politiques publiques au moment où cela est le plus porteur électoralement. Dans la période qui suit directement l'élection, les élus peuvent avoir intérêt à rendre visibles les effets de l'élection, encore fraîche dans l'esprit des électeurs. Ainsi, François Mitterrand avait promis de réaliser une grande partie de son programme dans les "cent jours " qui suivraient son élection à la présidence de la République. De même, de nombreuses observations de l'évolution des dépenses publiques au fil des cycles électoraux corroborent l'idée selon laquelle le parti au pouvoir a tout intérêt à convaincre les électeurs de son bilan, en étant actif dans les secteurs sur lesquels il s'était engagé durant l'année précédant l'élection - que ce soit

Premier ministre domine presque systématiquement les coalitions parlementaires. La seule exception est la période 1976-1981, lors de laquelle le nombre de députés RPR dépassait celui des députés UDF, parti du Premier ministre Raymond Barre. Cette période n'est pas incluse dans cette étude.

11. Cela revient à dire que l'influence des préférences du parti du Premier ministre est moins forte lorsque la gauche est au gouvernement, le Sénat français étant acquis à la droite depuis 1958. 
en termes de réduction des impôts ou d'augmentation des dépenses ${ }^{12}$. Notre troisième hypothèse, liée à la temporalité, peut donc se décomposer de la manière suivante :

H3a : L'influence des préférences du parti du Premier ministre sur l'agenda législatif est plus forte dans l'année qui suit une élection législative.

H3b : L'influence des préférences du parti du Premier ministre sur l'agenda législatif est plus forte dans l'année qui précède une élection législative.

\section{Les données du Comparative Agendas Project, un instrument privilégié de comparaison des agendas partisan et législatif}

L'analyse dynamique de l'influence de l'agenda partisan (autrement dit des priorités électorales du parti du futur Premier ministre) sur l'agenda législatif (les priorités législatives) exige de disposer de données comparables à ces deux niveaux. Les données collectées par le Comparative Agendas Project (CAP) nous semblent offrir une base particulièrement féconde à cet égard. Lancé à l'origine par $\mathrm{F}$. Baumgartner et $\mathrm{B}$. Jones pour comparer les agendas politiques américains, le CAP a évolué pour devenir un réseau international impliquant des chercheurs issus d'une quinzaine de pays, qui ont rassemblé des données relatives aux agendas législatifs, gouvernementaux, présidentiels, médiatiques, électoraux, etc ${ }^{13}$. Les documents sélectionnés - textes de lois, comptes rendus de conseil des ministres, vœux du président de la République, articles de presse, programmes électoraux ... - ont été, selon les cas, divisés en une ou plusieurs unités de codage - loi, article, phrase ou quasi-phrase. Chaque unité a été codée suivant une grille thématique classant les enjeux de politique publique en 28 catégories générales (macro-économie, santé, énergie, etc.) et plus de 250 sous-catégories (politique fiscale, organisation du système de soins, gestion des déchets nucléaires, etc. $)^{14}$. Cette procédure permet de restituer précisément l'évolution de la part d'attention consacrée à chaque enjeu au niveau de chacun des agendas étudiés.

La base de données du CAP présente trois avantages de taille pour étudier le lien entre compétition partisane et politiques publiques. Premièrement, elle propose des informations strictement comparables entre les deux niveaux, puisque les lois et les programmes électoraux sont codés en suivant une grille identique, qui rend compte de leur contenu de manière exhaustive et exclusive. Cela permet la définition de catégories transversales aux différents agendas et représente un avantage considérable par rapport aux autres données disponibles sur les positionnements partisans, notamment les données du Comparative Manifesto Project (CMP) et les enquêtes d'expert de Michael Laver ${ }^{15}$.

12. Les dépenses publiques tendent à augmenter dans les mois qui précèdent une élection. Voir par exemple R. J. Franzese (2002).

13. On pourra consulter les sites Internet suivants pour plus d'information: [http://www.policyagendas.org/] pour le cas américain; [http://www.comparativeagendas.org/] pour ce qui est de la comparaison européenne; [http://www.agendas-france.fr/] pour le cas français.

14. La grille de codage est disponible sur le site [www.agendas-france.fr]. Pendant les opérations de codage, des réunions mensuelles se sont déroulées afin de trancher des doutes éventuels, d'assurer la concordance entre les codeurs impliqués dans le traitement des différents agendas, et de vérifier la fiabilité des résultats de codage.

15. Comme ces dernières n'ont pas été élaborées dans l'objectif de comparer l'agenda partisan et l'agenda législatif, elles combinent des informations sur la saillance des différents enjeux (aspect rarement inclus dans les enquêtes d'expert mais essentiel dans le cadre du CMP), sur les instruments et les cibles envisagés par les politiques publiques prônées, leur cadrage, les positions adoptées, etc. De ce fait, les catégories utilisées ne sont pas toutes appropriées pour décrire les politiques publiques. Si l'on peut aisément attribuer certaines lois aux catégories "Éducation » ou « Droit et Sécurité ", il est plus difficile d'imaginer quel type de décision pourrait relever des catégories " Analyse marxiste ", " Décroissance » ou « Multiculturalisme », qui font partie de la grille de codage du CMP. 
Deuxièmement, la base de données du CAP couvre de nombreux pays, ce qui ouvre des perspectives considérables en matière de comparaison internationale. Ces possibilités sont d'autant plus essentielles que l'on creuse la question des conditions - notamment institutionnelles - dans lesquelles les partis modèlent les politiques publiques. Au-delà des comparaisons longitudinales, l'existence de données comparables entre pays ouvre la voie à des comparaisons entre systèmes fédéraux et centralisés, entre systèmes parlementaires et présidentiels, ou encore entre gouvernements unitaires et coalitions.

Enfin, la finesse inégalée de la grille de codage du CAP permet d'approfondir les observations en se concentrant sur les enjeux relevant d'un domaine particulier. Au sein de la catégorie " politique énergétique " par exemple, il devient possible de rendre compte des dynamiques d'attention aux différentes composantes que sont la politique nucléaire, les hydrocarbures, les énergies renouvelables, etc. Les comparaisons à large-échelle qui peuvent être réalisées grâce à ces données peuvent enfin être complétées par des études de cas facilitées par le format de la base, qui contient le texte intégral des programmes et le titre des lois codées. La lecture de ces textes permet d'appréhender d'autres dimensions que celle du niveau d'attention, comme par exemple le cadrage des enjeux et les positions adoptées par les partis et les législateurs.

Dans le cadre de cet article, nous souhaitons illustrer les potentialités du CAP à partir des données produites par le projet Agendas France sur les programmes électoraux et les lois promulguées en France, entre 1981 et 2009. Le pays et la période considérés se prêtent particulièrement bien à l'analyse de notre problématique. D'une part, l'impact partisan devrait être particulièrement élevé dans un régime comme celui de la $V^{\vee}$ République. Les régimes de type majoritaire, pour reprendre la classification développée par A. Lijphardt (1999), qui se caractérisent par un affrontement clair entre majorité et opposition, favorisent en effet l'influence partisane (Schmidt, 1996). D'autre part, la période sélectionnée s'ouvre sur l'élection de François Mitterrand à la présidence de la République, immédiatement suivie d'une victoire de la gauche aux élections législatives du mois de juin, qui marquent la première alternance de la $V^{\circledR}$ République. Depuis, la vie politique française a été rythmée par des alternances régulières et par trois périodes de cohabitation, entre 1986 et 1988, entre 1993 et 1995, puis entre 1997 et 2002. Ces configurations sont donc favorables pour identifier des changements liés au parti au pouvoir, mais aussi pour prendre la mesure de l'influence de la cohabitation sur l'empreinte politique du parti du Premier ministre.

La variable dépendante - l'agenda législatif - est opérationnalisée à partir de la base de données des « lois promulguées » entre 1978 et 2009, qui recense 2933 lois, dont 2602 approuvées par le Parlement durant la période qui nous intéresse. Cette base a été élaborée à partir du site internet du Sénat ${ }^{16}$, dont les informations ont été recoupées avec celles du site Légifrance ${ }^{17}$. Le codage thématique permet de connaître la part de l'agenda législatif consacrée à chacun des 28 secteurs de politiques publiques et des 250 sous-secteurs (nombre de lois promulguées dans un secteur/nombre total de lois promulguées) ${ }^{18}$.

Notre principale variable indépendante - les promesses du parti du futur Premier ministre est opérationnalisée à partir des données partisanes du Projet Agendas France, c'est-à-dire des données issues du codage des programmes présentés par les partis aux élections législatives.

16. [www.senat.fr].

17. [www.legifrance.gouv.fr].

18. Les analyses présentées dans cet article restituent les dynamiques d'attention aux différents secteurs et ne sont pas affinées en différenciant par sous-secteur. 
Bien entendu, travailler à partir des programmes électoraux laisse de côté les dynamiques propres aux campagnes électorales, qui ont lieu après que les programmes ont été rédigés. Cela ne permet pas non plus, de mesurer les réactions partisanes à l'évolution de la conjoncture en période non électorale. Cela étant, toute comparaison des positions partisanes doit être basée sur un corpus de documents uniformes, pouvant être considérés comme représentatifs de la position officielle et collectivement décidée des partis à un moment donné (voir Volkens, 2001 pour une argumentation plus exhaustive). C'est la raison pour laquelle ce travail s'appuie sur une démarche de codage de programmes électoraux.

L'unité de codage de ces productions textuelles est la quasi-phrase, qui contient un, et un seul argument et qui ne relève que d'un seul secteur de politiques publiques (Krippendorff, 2004). Pour la période considérée, nous avons analysé les programmes présentés par le principal vainqueur de chacune des sept élections législatives, qui comprennent un total de 5246 quasiphrases. Les données du CAP permettent d'inférer la part respective de chaque secteur dans le programme du parti du futur Premier ministre (nombre de quasi-phrases consacrées à un secteur/nombre total de quasi-phrases dans le programme).

Ces deux premières variables forment le cœur de notre modèle testant le lien entre préférences du parti du Premier ministre et priorités de l'agenda législatif. Afin de mieux déterminer les conditions dans lesquelles cette influence s'exerce, nous utilisons une série de variables supplémentaires, toutes dichotomiques. La première indique la présence ou l'absence de situation de cohabitation $(\mathrm{H} 2 \mathrm{a})$, la seconde précise si la majorité de l'Assemblée nationale diffère de celle du Sénat $(\mathrm{H} 2 \mathrm{~b})$ et les deux dernières distinguent la première et la dernière année de la mandature $(\mathrm{H} 3 \mathrm{a}$ et H3b). L'ensemble du modèle est contrôlé par une variable temporelle (année).

\section{Les politiques publiques françaises face à l'alternance entre 1981 et 2009}

La comparaison du poids relatif de chaque secteur de politiques publiques dans les agendas législatif et partisan est riche d'enseignements. Le tableau 1 représente ainsi la valeur moyenne (en \%) du poids des différents secteurs dans l'agenda législatif dans les gouvernements PS (VIIe,

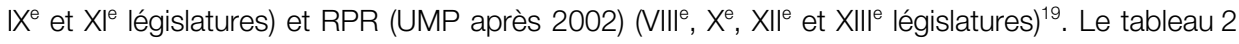
fait de même avec les programmes électorau $x^{20}$ des partis qui ont remporté les élections sur l'ensemble de la période étudiée ${ }^{21}$.

19. Les données originelles ont été pondérées par le nombre de lois promulguées au cours de chaque législature (nombre de lois approuvées dans chaque domaine pour une législature/nombre total de lois approuvées pendant cette législature).

20. Là encore, les pourcentages ont été pondérés par le nombre total de quasi-phrases dans chaque programme.

21. L'analyse a été limitée aux domaines politiques qui représentent une part significative des agendas partisans et législatifs. Dans les deux cas, les domaines "énergie " (code 8), "espace, science et technologie " (code 17), " domaine public et gestion de l'eau » (code 21) et " sport " (code 29) n'ont pas été étudiés, eu égard à leur saillance très faible sur toute la période étudiée. C'est également le cas de catégories telles que " accidents et incendies " ou " nécrologie ", qui ont été incorporées dans le codebook dans le cadre du codage d'articles de presse, mais qui ne sont pas utilisées pour coder les agendas qui nous intéressent ici. La catégorie "politique régionale" (code 24), qui n'est pas appropriée dans le cadre de l'étude de l'agenda législatif et qui est très peu représentée dans les données partisanes, a également été écartée, ainsi que la catégorie " autres " (code 99). La composition exacte de l'agenda du parti du futur Premier ministre et de l'agenda législatif de chaque législature est détaillée dans les tableaux 2 et 3 , en annexe. 
Tableau 1. Part moyenne des différents domaines de politiques publiques dans l'activité législative française, 1981-2009 (en \%) ${ }^{22}$

\begin{tabular}{|c|c|c|c|c|c|c|c|c|c|c|c|c|c|c|c|c|c|c|c|}
\hline \multirow{2}{*}{$\begin{array}{l}\text { Parti du } \\
\text { PM }\end{array}$} & \multicolumn{19}{|c|}{ Attention moyenne par domaine (en \%) } \\
\hline & 1 & 2 & 3 & 4 & 5 & 6 & 7 & 9 & 10 & 12 & 13 & 14 & 15 & 16 & 18 & 19 & 20 & 23 & Tot \\
\hline PS & 6,1 & 3,2 & 2,7 & 2,9 & 6,2 & 1,6 & 3,0 & 1,8 & 4,7 & 11,0 & 1,8 & 2,4 & 6,4 & 3,4 & 5,1 & 18,3 & 12,8 & 2,8 & 100 \\
\hline Écart-type & 1,6 & 1,7 & 0,9 & 1,8 & 1,9 & 1,1 & 1 & 0,6 & 1,5 & 0,4 & 0,7 & 0,7 & 3 & 1,2 & 2,7 & 8,6 & 1,5 & 1,1 & \\
\hline RPR-UMP & 6,1 & 3,9 & 2,5 & 1,5 & 6,9 & 1,4 & 2,0 & 2,2 & 5,7 & 12,2 & 1,3 & 2,6 & 6,1 & 4,1 & 4,0 & 17,8 & 11,7 & 2,8 & 100 \\
\hline Écart-type & 0,8 & 1,6 & 0,5 & 0,6 & 1,9 & 0,6 & 1,5 & 1,5 & 2,5 & 1,9 & 0,7 & 1,7 & 2,4 & 1,3 & 2,7 & 5,8 & 3,0 & 0,9 & \\
\hline
\end{tabular}

$\mathrm{N}=2602$

Source : Projet Agendas France.

NB: Les codes indiquent les domaines suivants: 1 = Macroéconomie, 2 = Droits de l'homme et discriminations, 3 = Santé, 4 = Agriculture, 5 = Travail et emploi, 6 = Éducation, 7 = Environnement, $9=$ Immigration, $10=$ Transports, 12 = Justice, 13 = Politique sociale, 14 = Politiques du logement, 15 = Régulations économiques, 16 = Défense, 18 = Commerce extérieur, 19 = Politique étrangère, 20 = Fonctionnement de l'État, 23 = Politique culturelle.

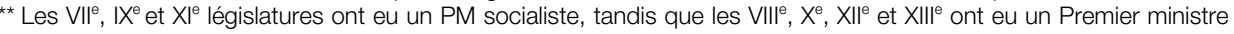
RPR ou UMP.

\section{Tableau 2. Part moyenne des différents domaines de politiques publiques dans les programmes du parti du futur PM, 1981-2009 (en \%)}

\begin{tabular}{|c|c|c|c|c|c|c|c|c|c|c|c|c|c|c|c|c|c|c|c|}
\hline \multirow[t]{2}{*}{ Partis } & \multicolumn{19}{|c|}{ Attention moyenne par domaine (en \%) } \\
\hline & 1 & 2 & 3 & 4 & 5 & 6 & 7 & 9 & 10 & 12 & 13 & 14 & 15 & 16 & 18 & 19 & 20 & 23 & Tot \\
\hline PS & 11,9 & 4,2 & 3,1 & 4,6 & 15,5 & 5,1 & 2,1 & 1,8 & 1,1 & 3,9 & 4,7 & 3,0 & 8,3 & 2,1 & 3,0 & 4,5 & 10,2 & 3,3 & 100 \\
\hline Écart-type & 6,4 & 1,7 & 2,9 & 0,1 & 5,7 & 2,5 & 1,6 & 1,7 & 0,5 & 4,9 & 0,6 & 0,6 & 7,7 & 2,1 & 2,0 & 3,4 & 2,1 & 2,2 & \\
\hline RPR-UMP & 13,8 & 2,4 & 4,7 & 2,4 & 11,3 & 10,1 & 2,3 & 4,6 & 1,3 & 9,8 & 6,6 & 3,3 & 3,6 & 1,5 & 2,5 & 4,4 & 10,2 & 2,7 & 100 \\
\hline Écart-type & 5,6 & 0,9 & 3,1 & 1,9 & 5,9 & 3,7 & 1,8 & 4,4 & 0,7 & 6,1 & 3,8 & 1,5 & 1,2 & 0,9 & 1,3 & 3,3 & 3,2 & 2,6 & \\
\hline
\end{tabular}

Source : Projet Agendas France.

NB : Les codes indiquent les domaines suivants: 1 = Macroéconomie, 2 = Droits de l'homme et discriminations, 3 = Santé, 4 = Agriculture, 5 = Travail et emploi, 6 = Éducation, 7 = Environnement, $9=$ Immigration, $10=$ Transports, 12 = Justice, 13 = Politique sociale, 14 = Politiques du logement, 15 = Régulations économiques, $16=$ Défense, 18 = Commerce extérieur, 19 = Politique étrangère, 20 = Fonctionnement de l'État, 23 = Politique culturelle.

De manière générale, la composition de l'agenda législatif paraît relativement homogène au cours des sept législatures étudiées. Les priorités, relativement stables au cours du temps, sont globalement les mêmes, que le Premier ministre soit issu du PS ou du RPR (UMP). Le tableau 1 révèle en effet la prédominance de cinq domaines sur l'agenda législatif, indépendamment de la couleur politique du Premier ministre : la " politique étrangère " (18,5\% en moyenne), le " fonctionnement de l'État » (12,2\%), la « justice » (11,6\%), le « travail et l'emploi » $(6,5 \%)$ et la « macro-économie » (6,1\%). Seules quelques différences méritent d'être soulignées à ce niveau agrégé. Les parlements majoritairement socialistes accordent une plus grande attention au secteur agricole - de manière plutôt contre-intuitive, le PS y consacre presque deux fois plus de lois que le RPR et l'UMP l'environnement -, $50 \%$ de lois en plus chez le PS ${ }^{23}$, la politique sociale, le commerce extérieur et le fonctionnement de l'État. Les législatures dominées par le RPR se caractérisent par une proportion légèrement supérieure de lois consacrées aux transports, à la justice et à la sécurité, ainsi qu'à la défense. Ces grandes lignes indiquent une tendance à la stabilité et vont dans le sens de l'hypothèse de l'inertie des agendas législatifs formulée par Baumgartner et Jones (1993).

22. Nous proposons une représentation graphique de ces données en annexe (graphique 2).

23. Notons toutefois une proportion relativement faible dans les deux cas. 
Mis en perspective avec le tableau précédent, le tableau 2 fait apparaître deux tendances principales. Premièrement, la hiérarchisation des problèmes politiques dans les programmes partisans ne reflète pas complètement celle que nous observons sur l'agenda législatif. La "politique étrangère ", qui fait l'objet d'une majorité de lois, n'est pas érigée en priorité dans les programmes électoraux. Tandis que les thèmes du «travail et de l'emploi », de la « macro-économie » et du « fonctionnement de l'État » sont importants dans les deux cas, leur saillance est sensiblement différente au niveau agrégé, chacun de ces thèmes faisant l'objet de 10 à $13 \%$ des propositions programmatiques, en moyenne.

Deuxièmement, la différentiation partisane est beaucoup plus nette qu'au niveau de l'agenda législatif. Certains contrastes ne sont pas surprenants au regard de la réputation des deux partis, à l'image de la surreprésentation des questions liées au travail, aux régulations économiques et aux libertés civiques dans les programmes du PS, et de la priorité plus importante accordée par le RPR-UMP aux questions d'immigration, de justice et de sécurité. En revanche, on s'attend peut-être moins au niveau d'attention significativement supérieur consacré par le PS à la politique agricole et, par le RPR-UMP, à l'éducation.

Le PS et l'UMP semblent mettre en avant des domaines distincts lors de leurs campagnes électorales. Si les activités législatives sont caractérisées par une stabilité et une convergence inter-partisane nettement plus marquées, les agendas ne sont pas nécessairement déconnectés si l'on désagrège les tendances observées au niveau de l'enjeu, ce que les régressions réalisées doivent permettre de préciser.

\section{«Do French Parties Matter? »}

Notre première hypothèse $(\mathrm{H} 1)$, selon laquelle les thèmes politisés par le parti du Premier ministre lors de la précédente campagne électorale tendent à être érigés en priorités sur l'agenda parlementaire, a été testée à l'aide d'une analyse de régression, dont les résultats sont reportés dans le tableau 3 ci-après.

\section{Tableau 3. L'influence des préférences partisanes des partis du PM sur l'agenda législatif (1981-2009)}

\begin{tabular}{lc}
\hline & Modèle 1 \\
\hline Agenda législatif & Beta \\
\hline Attention dans les programmes du parti du PM & .24 \\
& $(.08)^{\star \star \star}$ \\
Constante & 4.07 \\
$\mathrm{R}^{2}$ & $6 \%$ \\
$\mathrm{~N}$ & 126 \\
\hline
\end{tabular}

Source : Projet Agendas France.

${ }^{\star} p<0,10 ;{ }^{\star \star} p<0,05 ;{ }^{* \star \star} p<0,01$

Le tableau 3 indique clairement que les préférences du parti du Premier ministre influencent l'agenda législatif. Le lien entre agenda partisan et agenda législatif est statistiquement significatif $(p<.01)$ et la variable partisane permet d'expliquer $6 \%$ de la variance de l'agenda legislatif. L'effet marginal des préférences du parti du Premier ministre, quoique modeste, n'est pas négligeable. En moyenne, à l'augmentation d'un point de pourcentage de la saillance d'un domaine de politique publique dans le programme correspond une augmentation de l'attention à ce même domaine de 0,24 point dans l'agenda législatif. II semble dès lors intéressant d'explorer des pistes explicatives complémentaires 
pour expliquer les variations dans la mise sur agenda parlementaire des thèmes de prédilection du parti au gouvernement, et de préciser dans quelles conditions cette connexion est la plus forte.

\section{Le poids des institutions?}

Deux de nos hypothèses ont trait à l'impact de la configuration institutionnelle. D'une part, l'hypothèse H2a présuppose que l'influence des préférences du parti du Premier ministre sur l'agenda législatif est moins forte en période de cohabitation. D'autre part, l'hypothèse $\mathrm{H} 2 \mathrm{~b}$ affirme que l'influence des préférences du parti du Premier ministre sur l'agenda législatif est moins forte lorsque la majorité du Sénat diffère de celle de l'Assemblée nationale. Ces hypothèses ont été mises à l'épreuve dans le cadre d'un second modèle de régression intégrant de nouvelles variables explicatives, venant s'ajouter à la composition de l'agenda partisan, sous la forme de deux variables dichotomiques distinguant, d'une part, les périodes pour lesquelles le Sénat n'est pas de la même couleur que l'Assemblée nationale et, d'autre part, les situations de cohabitation. Les résultats des tests de ces deux hypothèses sont reproduits dans le tableau 4.

\section{Tableau 4. L'influence de la configuration institutionnelle sur} le lien entre agenda partisan et agenda législatif (1981-2009)

\begin{tabular}{lc}
\hline & Modèle 2 \\
\hline Agenda législatif & Beta \\
\hline Attention dans les programmes du parti du PM & .24 \\
Sénat hostile & $(.08)^{\star \star \star}$ \\
& 0.02 \\
Cohabitation & $\mathrm{NS}$ \\
& 0.12 \\
Constante & $\mathrm{NS}$ \\
$\mathrm{R}^{2}$ & 4.00 \\
$\mathrm{~N}$ & $6 \%$ \\
\hline
\end{tabular}

Source: Projet Agendas France.

${ }^{*} \mathrm{p}<0.10 ;{ }^{* \star} \mathrm{p}<0.05 ;{ }^{\star \star \star} \mathrm{p}<0.01$

Si le lien entre agenda partisan et agenda législatif reste valide et statistiquement significatif, l'inclusion de deux variables supplémentaires n'augmente pas le pouvoir explicatif du modèle. En fait, la variance expliquée se maintient à $6 \%$. Aucune des deux variables indépendantes considérées n'a d'effet significatif sur la composition de l'agenda législatif et nous ne sommes pas en mesure de corroborer nos hypothèses $\mathrm{H} 2 \mathrm{a}$ et $\mathrm{H} 2 \mathrm{~b}$ relatives aux obstacles inhérents à l'opposition du Sénat et du président de la République en période de cohabitation. Si des logiques d'opposition et de blocage existent, elles ne semblent pas se manifester au niveau de la mise sur agenda parlementaire. Ce résultat contredit certaines idées établies sur le fonctionnement de la $V^{e}$ République : la cohabitation ne semble pas empêcher la majorité parlementaire d'agir dans les domaines mis en avant dans son programme électoral ; les majorités de gauche à l'Assemblée nationale, systématiquement confrontées à un Sénat de droite, ne rencontrent pas plus de difficultés que les majorités de droite pour faire passer des lois dans leurs domaines de prédilection.

Ces résultats sont surprenants dans la mesure où ils vont à l'encontre de travaux précédents portant sur l'influence du Sénat sur l'activité législative (Grangé, 1981, 1984 ; Tardan, 1988 ; Tsebelis, Money, 1995). En particulier, ces recherches montraient que le Sénat est parvenu, de facto, à exercer un véritable pouvoir de veto que la Constitution ne lui reconnaît pas de jure. Cela s'expliquerait, d'une part, par la configuration institutionnelle du semi-présidentialisme français - le dispositif de 
cohabitation -, et, d'autre part, par l'absence d'alternance dans la composition de la Chambre Haute, dominée par les partis de centre-droit depuis la naissance de la V ${ }^{\circledR}$ République. Nos résultats semblent indiquer le contraire. Le Sénat demeure donc bien une chambre relativement impuissante s'agissant de la mise sur agenda parlementaire. Si la période de domination socialiste au Sénat, depuis septembre 2011, est trop courte pour être prise en compte dans ce travail, il sera intéressant de confirmer ou d'infirmer ces résultats lors des prochaines mandatures.

Nos résultats semblent donc indiquer que, dans le système institutionnel français, le travail de législation est celui de la majorité partisane telle qu'elle domine l'Assemblée nationale et le gouvernement.

\section{L'influence des cycles électoraux?}

Notre troisième et dernier ensemble d'hypothèses a trait au temps politique et à l'influence des cycles électoraux sur la capacité du parti au pouvoir à influencer l'agenda législatif. L'hypothèse H3a suppose que l'influence des préférences du parti du Premier ministre sur l'agenda législatif soit plus forte dans l'année qui suit une élection législative. D'autre part, selon l'hypothèse H3b, l'influence des préférences du parti du Premier ministre sur l'agenda législatif est plus forte dans l'année qui précède une élection législative. Le test de ces hypothèses apparaît dans le graphique 1 qui montre l'évolution de la corrélation entre la proportion d'attention consacrée à chaque enjeu sur l'agenda partisan et la proportion d'attention consacrée aux mêmes enjeux sur l'agenda législatif.

\section{Graphique 1. Corrélation entre agenda partisan et agenda législatif $(1981-2009)^{24}$}

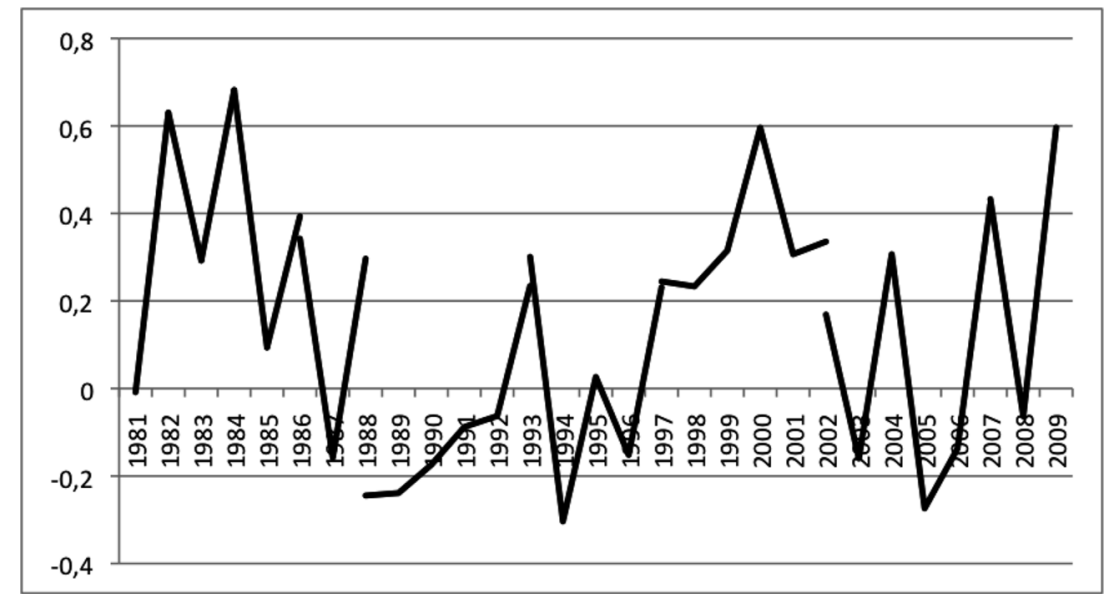

Source : Projet Agendas France.

Le graphique 1 révèle des fluctuations importantes du lien entre agenda partisan et agenda parlementaire. La corrélation entre les deux varie considérablement, pour passer de valeurs négatives entre 1988 et 1996, puis entre 2003 et 2008 aux valeurs record de plus de 0,6 en 1982, 1984, 2000 et 2009. S'il paraît difficile, à première vue, de tirer des conclusions générales à propos de l'évolution des priorités parlementaires au cours des cycles électoraux, on peut noter l'augmentation -

24. Les valeurs de corrélation correspondant aux années d'une même législature sont représentées par une ligne continue. Chaque année électorale donne lieu à deux valeurs distinctes : la corrélation entre les priorités affichées par le parti du Premier ministre sortant avec l'agenda législatif de la période pré-électorale et la corrélation entre l'agenda du parti du nouveau Premier ministre avec celui du parlement, entre la date des élections et la fin de l'année civile. L'année 1986 a, par exemple, été divisée en deux périodes : la première débute le $1^{\text {er }}$ janvier et prend fin le 16 mars, date des élections. La seconde couvre le reste de l'année et se termine donc le 31 décembre. 
systématique, mais plus ou moins prononcée selon les cas - de la corrélation entre agenda législatif et agenda partisan à l'approche des élections, ce qui tend à corroborer notre hypothèse H3b.

Les résultats sont moins nets s'agissant de la première année des cycles électoraux, du fait de différences entre les législatures dominées par le PS et par le RPR (I'UMP). Les majorités conduites par un Premier ministre de droite se comportent conformément à l'hypothèse H3a et $\mathrm{H} 3 \mathrm{~b}$ et placent leurs enjeux de prédilection sur l'agenda parlementaire en début et en fin de cycle, avec un agenda législatif moins représentatif des thèmes affichés dans les programmes électoraux en milieu de cycle. En revanche, lorsque le Premier ministre est socialiste, les lois adoptées juste après l'élection ne reflètent pas les enjeux électoraux placés au centre du programme du PS, mais le lien entre les deux agendas tend à se renforcer tout au long du cycle. Ce résultat demanderait à être approfondi en s'intéressant au profil des thèmes placés sur l'agenda par chaque gouvernement, afin d'analyser les contraintes " externes » qui pèsent sur la mise sur agenda de certains enjeux, mais aussi la manière dont les membres du gouvernement et du parlement hiérarchisent leurs objectifs, entre policy, office et vote.

\section{Conclusion}

Notre revue de la littérature montre que les politistes en savent finalement assez peu sur la nature de l'influence exercée par les partis sur les politiques publiques. La sociologie française de l'action publique réserve une place sans doute trop modeste aux partis politiques, tandis que le débat anglosaxon du «Do parties matter? » est loin d'avoir fait le tour du sujet, du fait de sa focalisation exclusive sur des indicateurs macro-budgétaires. Nous plaidons pour une opérationnalisation plus fine des variables dépendantes et indépendantes ainsi que pour une différentiation de la problématique vers une interrogation relative aux conditions dans lesquelles les partis marquent les politiques publiques.

Pour traiter cette question au prisme de la dimension de la mise sur agenda, les bases de données collectées et constamment élargies par le CAP constituent un fondement empirique sans précédent, qui permettra de comparer l'ordre du jour de nombreuses arènes - partisanes et parlementaires notamment - dans plusieurs pays aux caractéristiques variées.

Nous avons tenté d'illustrer ces potentialités en partant d'une série d'hypothèses, que nous avons testées dans le cadre d'une étude centrée sur les agendas partisan et législatif français, entre 1981 et 2009. Ancrée au niveau macro, notre étude confirme l'inertie de l'agenda parlementaire, dont les priorités sont très stables au fil du temps, et l'absence de relation forte et systématique entre les thèmes électoraux affichés par le parti du Premier ministre et l'agenda parlementaire. Nos résultats sont ainsi cohérents avec d'autres études de cas - fondées elles aussi sur les données CAP notamment dans les cas italien (Borghetto, Carammia, Zucchini, 2011), britannique (Bevan, John, Jennings, 2011), danois (Green-Pedersen, 2010) ou espagnol (Chaqués, Palau, 2011).

Notre étude met toutefois en évidence une influence, modeste, des partis sur l'agenda législatif, qui se traduit en particulier par l'adoption d'un plus grand nombre de lois dans les domaines du fonctionnement de l'État, de l'environnement, des politiques sociales et de l'agriculture, lorsque le PS est au pouvoir. Lorsque c'est le RPR (puis l'UMP) qui est aux affaires, une attention accrue est dévouée à la justice, aux transports et à la défense. L'empreinte partisane sur l'agenda parlementaire est particulièrement forte en fin de cycle électoral mais aussi, dans le cas des gouvernements de droite, dans l'année qui suit une élection. II semble donc que le temps politique pèse sur les arbitrages effectués entre policy, office et vote. Face à la tension qui existe entre la tendance à l'inertie 
et à l'équilibre ponctué qui caractérise l'agenda parlementaire, les membres du gouvernement semblent faire plus d'efforts pour imposer leurs thèmes de prédilection à l'ordre du jour dans les mois qui suivent leur élection et à l'approche de la prochaine élection. En revanche, l'influence des partis sur les politiques ne semble pas significativement altérée en cas d'opposition du Sénat ou du président de la République. Ni la cohabitation, ni le fait que les gouvernements de gauche soient en opposition avec le Sénat ne réduisent la force du lien entre agenda partisan et agenda parlementaire.

Dès lors, ces analyses permettent, en contrepoint, d'ouvrir de nombreuses pistes de recherche. La variable dépendante pourrait être appréhendée de façon plus fine en étendant l'analyse à l'agenda gouvernemental| ${ }^{25}$. Une étude de cas centrée sur la France entre 1980 et 2009 a montré qu'il existe des différences entre ces deux agendas, et que l'agenda législatif semble plus sensible aux préférences des partis (Froio, 2010). Des indicateurs alternatifs à celui de la part de chaque secteur de politique publique pourraient en outre être envisagés, comme par exemple celui de la hiérarchisation de ces secteurs dans l'agenda législatif et gouvernemental ${ }^{26}$.

La démarche consistant à reformuler la question du "Do Parties Matter? », en s'interrogeant sur les conditions dans lesquelles l'influence partisane s'exerce, devra être enrichie en suivant plusieurs pistes comparatives. D'une part, la question de la configuration institutionnelle et du nombre de veto players doit être creusée en comparant plusieurs pays présentant des systèmes politiques différents. D'autre part, le pouvoir explicatif de la visibilité des problèmes politiques appelle une comparaison entre plusieurs enjeux, intégrant des dimensions supplémentaires telles que la médiatisation des campagnes électorales et des problèmes publics.

Les données du CAP offrent un fondement empirique utile pour progresser dans ces deux voies. Afin de mieux cerner les mécanismes causaux à l'œuvre, les analyses quantitatives de ces données devront être complétées par des études de cas plus qualitatives, mieux à même de restituer des aspects tels que l'influence du ou des partenaires de coalition, la portée relative de facteurs explicatifs non partisans, mais aussi et surtout les changements politiques qui prennent d'autres formes que celle de la mise sur agenda des enjeux de politique publique.

Simon Persico

Centre d'études européennes, Sciences Po simon.persico@sciences-po.org

Caterina Froio

Department of Political and Social Sciences Institut universitaire européen caterina.froio@eui.eu

Isabelle Guinaudeau

Department of Political and Social Sciences Institut universitaire européen Isabelle.guinaudeau@eui.eu

25. II est difficile de prendre en compte les politiques publiques qui ne font pas l'objet de lois dans ce type d'étude, dans la mesure où leur recensement exhaustif est impossible.

26. Voir S. Brouard et al. (2010) pour un argumentaire en faveur de cet indicateur. 


\section{Annexes}

\section{Codes du Projet Agendas France}

\begin{tabular}{ll}
\hline 1. Macroéconomie & 16. Défense \\
2. Droits de l'homme et discriminations & 17. Espace, science et technologie \\
3. Santé & 18. Commerce extérieur \\
4. Agriculture & 19. Politique étrangère \\
5. Travail et emploi & 20. Fonctionnement de l'État \\
6. Éducation & 21. Domaine public et gestion de l'eau \\
7. Environnement & 23. Politique culturelle \\
8. Énergie & 24. Politique locale et régionale \\
9. Immigration & 25. Risques et catastrophes naturels et \\
10. Transports & \multicolumn{1}{c}{ météorologiques } \\
12. Justice & 28. Accidents et incendies \\
13. Politique sociale & 29. Sport \\
14. Logement & 30. Nécrologie \\
15. Régulations économiques & 31. Églises et religions \\
& 99. Autres
\end{tabular}

Source : Projet Agendas France.

Tableau 5. Composition de l'agenda législatif en France, 1981-2009 (en $\%$ et par législature)

\begin{tabular}{lccccccc}
\hline Domaines de politiques publiques & \multicolumn{7}{c}{ Législature } \\
& VII & VIII & IX & X & XI & XII & XIII \\
\hline Macroéconomie & 7,9 & 6,7 & 4,9 & 6,6 & 5,5 & 4,9 & 6,2 \\
Droits de l'homme et discriminations & 2,7 & 3,7 & 1,8 & 2,3 & 5 & 3,4 & 6,2 \\
Santé & 2,3 & 3 & 3,6 & 2,3 & 1,9 & 1,9 & 2,7 \\
Agriculture & 4,8 & 1,2 & 2,7 & 2,3 & 1,2 & 1 & 1,3 \\
Travail et emploi & 8,1 & 9,8 & 6,2 & 5,8 & 4,3 & 6,2 & 5,9 \\
Éducation & 2,5 & 0,62 & 1,8 & 1,5 & 0,4 & 1,9 & 1,7 \\
Environnement & 2,9 & 0 & 4 & 2,9 & 2,1 & 3,4 & 1,7 \\
Immigration & 1,3 & 0,6 & 1,3 & 4,2 & 2,4 & 2,3 & 1,7 \\
Transports & 6,1 & 6,7 & 4,9 & 2,3 & 3,1 & 5,5 & 8,3 \\
Justice & 10,6 & 13,5 & 11,4 & 9,5 & 11,1 & 13,5 & 12,2 \\
Politique sociale & 2,1 & 1,8 & 2,25 & 0,7 & 0,9 & 1,9 & 0,7 \\
Logement & 3,2 & 4,9 & 1,8 & 2,6 & 2,1 & 1,7 & 1 \\
Régulations économiques & 7,1 & 5,5 & 8,9 & 5,8 & 3,1 & 3,8 & 9,4 \\
Défense & 3,4 & 5,5 & 2,2 & 2,6 & 4,5 & 3,6 & 4,8 \\
Espace, science et technologie & 2,1 & 0,6 & 1,5 & 0,7 & 0,9 & 2,1 & 3,4 \\
Commerce extérieur & 3,2 & 2,4 & 4 & 7,9 & 8,2 & 3,8 & 2 \\
Politique étrangère & 10,4 & 10,4 & 17 & 20,9 & 27,5 & 23,6 & 16,3 \\
Fonctionnement de l'État & 13,9 & 15 & 13,4 & 13,4 & 11,1 & 8,3 & 10,1 \\
Culture & 3,6 & 3,7 & 3,3 & 2,6 & 1,6 & 3,23 & 1,7 \\
N & $\mathbf{5 1 7}$ & $\mathbf{1 6 2}$ & $\mathbf{4 4 5}$ & $\mathbf{3 7 8}$ & $\mathbf{4 1 4}$ & $\mathbf{4 6 5}$ & $\mathbf{2 8 7}$ \\
\hline
\end{tabular}

Source : Projet Agendas France. 
Tableau 6. Composition de l'agenda des partis des PM en France, 1981-2007

\begin{tabular}{|c|c|c|c|c|c|c|c|}
\hline \multirow[b]{2}{*}{$\begin{array}{l}\text { Domaines de } \\
\text { politiques publiques }\end{array}$} & \multicolumn{7}{|c|}{ Année électorale/Parti du PM } \\
\hline & \multicolumn{7}{|c|}{$\%$ d'attention } \\
\hline Macroéconomie & 10,5 & 21,2 & 23 & 14,7 & 14,02 & 11,3 & 8,07 \\
\hline Droits de l'homme et discriminations & 6,22 & 2,62 & 3,41 & 1,2 & 3,04 & 2,26 & 3,46 \\
\hline Santé & 5,25 & 5,03 & 0,8 & 0,17 & 6,3 & 6,7 & 6,93 \\
\hline Agriculture & 4,66 & 1,96 & 4,71 & 5,15 & 4,47 & 0,9 & 1,61 \\
\hline Travail et emploi & 14,59 & 18,8 & 21,58 & 4,63 & 10,3 & 12,21 & 9,73 \\
\hline Éducation & 6,22 & 5,03 & 2,3 & 13,74 & 6,91 & 10,85 & 10,87 \\
\hline Environnement & 3,69 & 1,53 & 0,4 & 0,34 & 2,23 & 2,62 & 4,55 \\
\hline Énergie & 2,92 & 0,21 & 0,1 & 0,17 & 1,01 & 0,9 & 0,99 \\
\hline Immigration & 1,55 & 4,81 & 0,2 & 10,6 & 3,65 & 0,45 & 2,7 \\
\hline Transports & 1,16 & 0,21 & 1,5 & 1,37 & 0,6 & 1,8 & 1,75 \\
\hline Justice & 1,75 & 4,59 & 0,3 & 14,4 & 9,5 & 15,8 & 4,55 \\
\hline Politique sociale & 5,44 & 1,75 & 4,31 & 5,49 & 4,47 & 8,59 & 10,4 \\
\hline Logement & 2,52 & 3,06 & 3,71 & 1,37 & 2,84 & 3,61 & 5,12 \\
\hline Régulations économiques & 3,89 & 5,03 & 17,2 & 2,5 & 3,86 & 4,07 & 2,89 \\
\hline Défense & 3,89 & 1,75 & 0,5 & 0,17 & 0 & 1,8 & 2,37 \\
\hline Espace, science et technologie & 0,19 & 0,43 & 0,6 & 0,51 & 0,4 & 1,35 & 0,94 \\
\hline Commerce extérieur & 1,36 & 3,06 & 5,32 & 2,23 & 2,43 & 0,9 & 3,89 \\
\hline Politique étrangère & 4,28 & 2,4 & 1,2 & 2,06 & 7,92 & 4,07 & 9,11 \\
\hline Fonctionnement de l'État & 11,47 & 8,75 & 7,83 & 14,94 & 11,38 & 8,59 & 8,54 \\
\hline Culture & 4,66 & 6,56 & 0,8 & 1,37 & 4,47 & 1,35 & 1,37 \\
\hline Politique régionale & 3,69 & 1,09 & 0 & 2,57 & 0 & 0 & 0 \\
\hline $\mathrm{N}$ & 514 & 457 & 996 & 582 & 492 & 221 & 2106 \\
\hline
\end{tabular}

Source : Projet Agendas France.

\section{Graphique 2. Attention moyenne aux différents domaines de politiques publiques dans les programmes du PS et du RPR-UMP (1981-2007)}

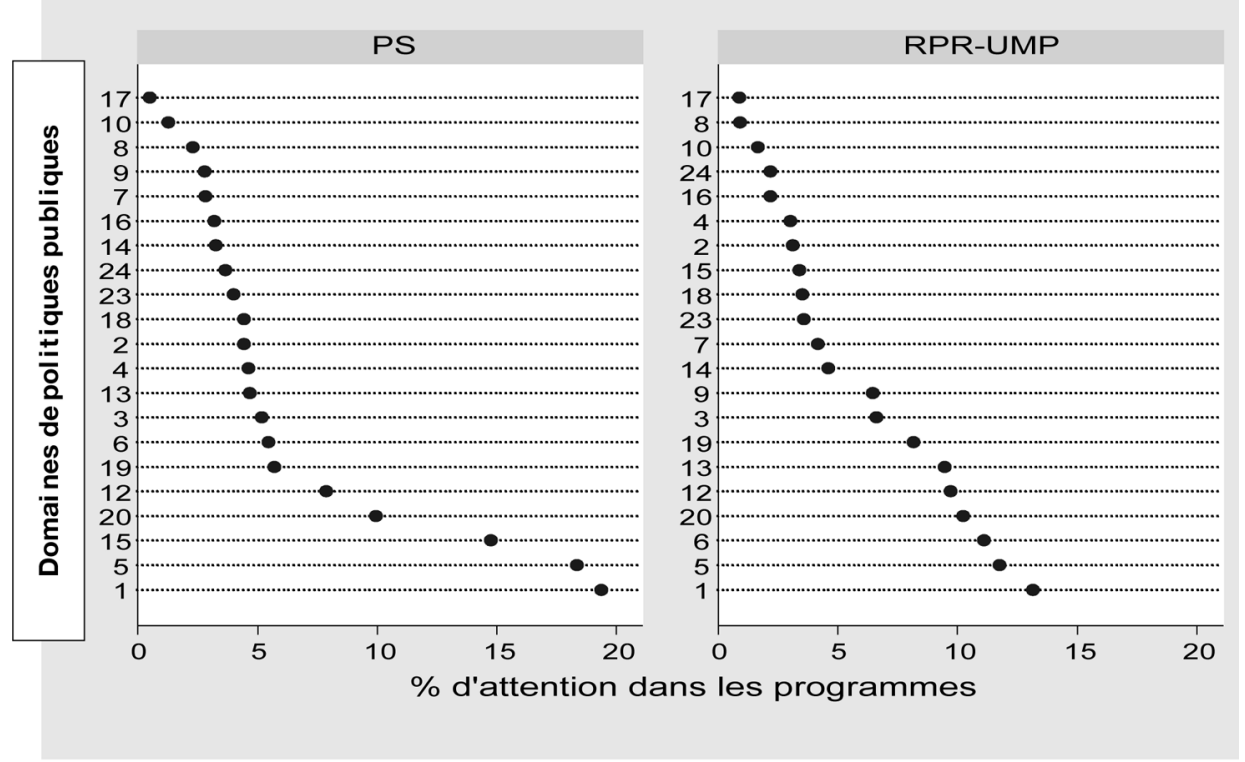

Source : Projet Agendas France.

Les axes verticaux des graphiques supra représentent les catégories de politiques publiques du Projet Agendas France, qui sont classées par ordre d'importance croissante dans les programmes du PS (à gauche) et du RPR (de l'UMP) à droite. Dans la mesure où la saillance de ces domaines est différente dans les programmes des deux partis, l'ordre des domaines n'est pas le même dans les deux graphiques. 


\section{Bibliographie}

AARDAL, B., VAN WIJNEN, P. (2005), "Issue Voting ", in Thomassen, J. (ed.), The European Voter, Oxford, Oxford University Press, p. 192-213.

ADLER, E. S., WILKERSON, J. (2008), «Intended Consequences: Juridictional Reform and Issue Control in the US House of Representatives ", Legislative Studies Quarterly, 33, p. 85-112.

BALDINI, G., PAPPALARDO, A. (2009), Elections, Electoral Systems and Volatile Voters, Basingstoke, Palgrave MacMillan.

BALE, T., HAMPSHIRE, T., PARTOS, R. (2011), "Having One's Cake and Eating It Too: Cameron's Conservatives and Immigration ", Political Quarterly, 82, p. 398-406.

BARTHE, Y. (2006), Le Pouvoir d'indécision? La mise en politique des déchets nucléaires, Paris, Economica.

BAUMGARTNER, F. R. (2004), "Political Agendas", dans International Encyclopedia of the Social \& Behavioral Sciences, Amsterdam, Elsevier, p. 288-290.

BAUMGARTNER, F. R., BROUARD, S., GROSSMAN, E. (2009), "Agenda-Setting Dynamics in France: Revisiting the Partisan Hypothesis ", French Politics, 7 (2), p. 75-95.

BAUMGARTNER, F. R., DE BOEF, S., BOYDSTUN, A. (2008), The Decline of the Death Penalty and the Discovery of Innocence, Cambridge, Cambridge University Press.

BAUMGARTNER, F. R., GREEN-PEDERSEN, C., JONES, B. D. (2006), "Comparative Studies of Policy Agendas ", Journal of European Public Policy, 13 (7), p. 959-974.

BAUMGARTNER, F. R., GREEN-PEDERSEN C., JONES, B. D. (eds) (2008), Comparative Studies of Policy Agendas, Londres, Routledge.

BAUMGARTNER, F. R., JONES, B. D. (1993), Agendas and Instability in American Politics, Chicago (III.), University of Chicago Press.

BAUMGARTNER, F. R., JONES, B. D. (2002), Policy Dynamics, Chicago (III.), University of Chicago Press.

BAUMGARTNER, F., JONES, B. (2005), The Politics of Attention: How Government Prioritizes Problems, Chicago (III.), University of Chicago Press.

BÉLANGER, É., MEGUID, B. (2008), «Issue Salience, Issue Ownership, and Issue-Based Vote Choice ", Electoral Studies, 27 (3), p. 477-491.

BEVAN, S., JOHN, P., JENNINGS, W. (2011), "Keeping Party Programmes on Track: The Transmission of the Policy Agendas of Executive Speeches to Legislative Outputs in the United Kingdom ", European Political Science Review, 3 (3), p. 395-417.

BEYME, K. VON (1985), Political Parties in Western Democracies, New York (N. Y.), St. Martin's Press.
BEZES, P., SINÉ, A. (dir.) (2011), Gouverner (par) les finances publiques, Paris, Presses de Sciences Po.

BLAIS, A. (2003), «Les élections affectent-elles les politiques gouvernementales? Le cas des dépenses publiques", Revue française de science politique, 53 (6), p. 929-940.

BLAIS, A., BLAKE D., DION, S. (1993), «Do Parties Make a Difference? Parties and the Size of Government in Liberal Democracies ", American Journal of Political Science, 37 (1), p. 40-62.

BLAIS, A., BLAKE D., DION, S. (1996), "Do Parties Make a Difference? A Reappraisal ", American Journal of Political Science, 40 (2), p. 514-520.

BOIX, C. (2000), "Partisan Governments, the International Economy, and Macroeconomic Policies in Advanced Nations, 1960-93 ", World Politics, 53 (1), p. 38-73.

BORGHETTO, E., CARAMANIA, M., ZUCCHINI, F. (2010), "The Impact of Party Policy Priorities on Italian Law-Making from the First to the Second Republic (1987-2006) ", communication à l'Internal Seminar of the Center for American Politics and Public Policy, University of Washington.

BOUSSAGUET, L. (2008), La Pédophilie, problème public ? : France, Belgique, Angleterre, Paris, Dalloz.

BROUARD, S. (2009), "The Politics of Constitutional Veto in France?: Constitutional Council, Legislative Majority and Electoral Competition ", West European Politics, 32 (2), p. 384-403.

BROUARD, S., GROSSMAN, E., GUINAUDEAU, I. (2010), "Issue Competition from Issue Ownership to Issue Uptake. The Issue Content of Party Competition in France ", communication à la Comparative Agendas Project annual conference, Seattle (Or.), 19 juin.

BROUARD, S., GUINAUDEAU, I. (2011), "Nuclear Policy in France. High Profile Policy and Low Salient Politics ", communication au workshop The Comparative Politics and Policies of Nuclear Energy in Western Europe, Munich, 3 juin.

BROWN, M., COTE, O. JR, LYNN-JONES, S. M. (2000), America's Strategic Choice. Revised Edition, Cambridge (Mass.), MIT Press.

BUDGE, I., HOFFERBERT, R. I. (1990), « Mandates and Policy Outputs: US Party Platforms and Federal Expenditures ", American Political Science Review, 84 (1), p. 111-131.

BUDGE, I., KLINGEMANN, H.-D., VOLKENS, A., et al. (eds) (2001), Mapping Policy Preferences: Estimates for Parties, Electors, and Governments, 1945-1998, Oxford, Oxford University Press.

BUDGE, I., MCDONALD, M. D. (2006), « Choices Parties Define: Policy Alternatives in Representative Elections, 17 Countries 1945-1998 ", Party Politics, 12 (4), p. 451-466. 
BUDGE, I., ROBERTSON, D., HEARL, D. J. (1987), Ideology, Strategy and Party Change: Spatial Analyses of Post-War Election Programmes in 19 Democraties, Cambridge, Cambridge University Press.

BURSTEIN, P., LINTON, A. (2002), " The Impact of Political Parties, Interest Groups, and Social Movement Organizations on Public Policy: Some Recent Evidence and Theoretical Concerns ", Social Forces, 81 (2), p. 381-408.

CAMERON, D. R. (1978), «The Expansion of the Public Economy: A Comparative Analysis ", American Political Science Review, 72 (4), p. 1243-1261.

CARMINES, E. G, STIMSON, J. A. (1989), Issue Evolution: Race and the Transformation of American Politics, Princeton (N. J.), Princeton University Press.

CASTLES, F. G. (1982), The Impact of Parties: Politics and Policies in Democratic Capitalist States, Londres, Sage Publications.

CHAQUÉS, L., PALAU, A. (2011). «Are Spanish Policy Makers Responding to Citizens? ", West European Politics, 34 (4), p. 871-894.

COBB, R. W., ELDER, C. D. (1971), "The Politics of Agenda-Building: An Alternative View for Modern Democratic Theory ", Journal of Politics, 33 (4), p. 892-915.

COBB, R. W., ELDER, C. D. (1972), Participation in American Politics: the Dynamics of Agenda-Building, Boston (Mass.), Allyn and Bacon.

COMAN, R., PERSICO, S. (2010), "Politiques publiques et partis politiques ", dans BOUSSAGUET, L., JACQUOT, S., RAVINET, P. (dir.), Dictionnaire des politiques publiques, Paris, Presses de Sciences Po, p. $482-491$.

CONLEY, R. S., BEKAFIGO, M. (2006), « The Determinants of Legislative Productivity: Parties, Coalitions, and "Divided Government" in the US, France, and Ireland ", communication au $32^{\mathrm{e}}$ congrès annuel de l'American Political Science Association, Philadelphia.

COS, R. (2011), "Pour une sociologie du désengagement électoral. Le Parti socialiste et les privatisations du gouvernement Jospin ", communication au Xle Congrès de l'Association française de science politique, Strasbourg.

CROUCH, C. (2004), Post-democracy, Cambridge, Polity Press.

CULPEPPER, P. D. (2011), Quiet Politics and Business Power: Corporate Control in Europe and Japan, New York (N. Y.), Cambridge University Press.

CUTRIGHT, P. (1965), "Political Structure, Economic Development, and National Social Security Programs ", American Sociological Review, 70 (5), p. 537-550.

DAVIS, O., DEMPSTER, M. H., WILDAVSKY, A. (1966), "A Theory of the Budgeting Process ", American Political Science Review, 60, p. 529-547.

DOWNS, A. (1957), An Economic Theory of Democracy, New York (N. Y.), Harper.

ÉVRARD, A. (2010), Intégrer les énergies renouvelables aux politiques publiques de l'électricité en Europe. Une comparaison ? Allemagne, Danemark, France, thèse de doctorat, Paris, Institut d'études politiques.

FAUCHER-KING, F., LE GALÈS, P. (2007), Tony Blair 1997-2007. Le bilan des réformes, Paris, Presses de Sciences Po.

FRANZESE, R. J. (2002), "Electoral and Partisan Cycles in Economic Policies and Outcomes ", Annual Review of Political Science, 5 (1), p. 369-421.

FROIO, C. (2010), L'Influence des partis sur l'attention gouvernementale. Une étude du rôle des partis de gouvernement en France entre 1979 et 2009, mémoire de master 2, Paris, Institut d'études politiques.

GARRAUD, P. (2010), "Agendas/Émergence ", dans BOUSSAGUET, L., JACQUOT, S., RAVINET, P. (dir.), Dictionnaire des politiques publiques, Paris, Presses de Sciences Po, p. 234-241.

GAXIE, D. (2003), La Démocratie représentative, Paris, Montchrestien.

GRANGÉ, J. (1981), « Attitudes et vicissitudes du Sénat (1958-1980) ", Revue française de science politique, 31 (1), p. 32-84.

GRANGÉ, J. (1984), « L'efficacité normative du Sénat », Revue française de science politique, 34 (4), p. 955-987.

GREEN-PEDERSEN, C. (2010), "New Issues, New Cleavages, and New Parties. How to Understand Change in West European Party Competition ", communication à la Conference of the Comparative Agendas Project, Seattle.

GREEN-PEDERSEN, C., BLOMQVIST, P. (2004), «Defeat at Home? Issue-Ownership and Social Democratic Support in Scandinavia ", Government and Opposition, 39 (4), p. 587-613.

GREEN-PEDERSEN, C., MORTENSEN, P. (2007), "Issue-Competition, Issue-Ownership and Political Parties ", communication à la $23^{\ominus}$ conférence annuelle de la Midwest Political Science Association, Chicago.

GREEN-PEDERSEN, C., WILKERSON, J. (2006), "How Agenda-setting Attributes Shape Politics: Problem Attention, Agenda Dynamics and Comparative Health Policy Developments in the US and Denmark ", Journal of European Public Policy, 13 (7), p. 1039-1052.

GROSSMAN, E. (2009), "The President's Choice? Government and Cabinet Turnover under the Fitfth Republic ", West European Politics, 32(2), p. 268-286.

GRUNBERG, G., HAEGEL, F. (2007), La France vers le bipartisme: la présidentialisation du PS et de l'UMP, Paris, Presses de Sciences Po.

GUINAUDEAU, I. (2011), L'européanisation de la compétition électorale en France, en Allemagne et au Royaume-Uni (1986-2009), thèse de doctorat, Sciences Po Bordeaux.

HALL, P. A., TAYLOR R. C. R. (1996), « Political Science and the Three New Institutionalisms ", Political Studies, 44 (5), p. 936-957.

HANIFF, G. M. (1976), "Politics, Development and Social Policy: A Cross-National Analysis ", European Journal of Political Research, 4 (4), p. 361-376. 
HASSENTEUFEL, P. (2010), «Les processus de mise sur agenda? Sélection et construction des problèmes publics ", Informations sociales (157), p. 50-58.

HASSENTEUFEL, P., SMITH, A. (2002), «Essoufflement ou second souffle? L'analyse des politiques publiques "à la française" ", Revue française de science politique, 52 (1), p. 53-73.

HÄUSERMANN, S. (2010), "Solidarity with Whom? Why Organized Labour is Losing Ground in Continental Pension Politics ", European Journal of Political Research, 49 (2), p. 233-256.

HECLO, H. (1974), Modern Social Politics in Britain and Sweden, New Haven (Conn.), Yale University Press.

HIBBS, D. A. (1977), "Political Parties and Macroeconomic Policy ", American Political Science Review, 71 (4), p. 1467-1487.

HIBBS, D. A. (1987), The Political Economy of Industrial Democracies, Cambridge (Mass.), Harvard University Press.

IMBEAU, L. M., PÉTRY, F., LAMARI, M. (2001), « LeftRight Party Ideology and Government Policies: A MetaAnalysis ", European Journal of Political Research, 40 (1), p. 1-29.

JOBERT, B. (1994), Le Tournant néo-libéral en Europe : idées et recettes dans les pratiques gouvernementales, Paris, L'Harmattan.

KATZ, R. S., MAIR (1995), " Changing Models of Party Organization and Party Democracy : the Emergence of the Cartel Party ", Party politics, 1 (1), p. 5-28.

KEOHANE, R. O., MILNER, H. V. (1996), Internationalization and Domestic Politics, Cambridge, Cambridge University Press.

KINGDON, J. W. (1984), Agendas, Alternatives, and Public Policies, New York (N. Y.), Longman.

KIRCHHEIMER, O. (1966), "The Transformation of the Western European Party Systems ", in KIRCHHEIMER, O. (ed.), Political Parties and Political Development, Princeton (N. J.), Princeton University Press, p. 177-200.

KITSCHELT, H. (1999), "European Social Democracy between Political Economy and Electoral Competition ", in KITSCHELT, H. et al. (eds), Continuity and Change in Contemporary Capitalism, Cambridge, Cambridge University Press, p. 317-345.

KLEINNIJENIHUIS, J., RIETBERG, E., (1995), "Parties, Media, the Public and the Economy: Patterns of Societal Agenda-Setting ", European Journal of Political Research, 28 (1), p. 95-118.

KLINGEMANN, H.-D., HOFFERBERT, R. I., BUDGE, I. (1994), Parties, Policies, and Democracy, Boulder (Colo.), Westview Press.

KLINGEMANN, H.-D., VOLKENS, A., BARA, J. (eds) (2006), Mapping Policy Preferences II? Estimates for Parties, Electors, and Governments in Eastern Europe, European Union, and OECD 1990-2003, Oxford, Oxford University Press.

KORPI, W., PALME, J. (2003), « New Politics and Class Politics in the Context of Austerity and Globalization:
Welfare State Regime", American Political Science Review, 97, p. 425-446.

KRIPPENDORFF, K. (2004), Content Analysis: an Introduction to its Methodology, Thousand Oaks (Calif.), Sage.

LASCOUMES, P. (2009), « Les compromis parlementaires, combinaisons de surpolitisation et de sous-politisation ", Revue française de science politique, 59 (3), p. 455-479.

LAZARDEUX, S. (2008), The Effects of Divided Government on Legislative Productivity in France, $\mathrm{PhD}$ thesis, Washington (D. C.), University of Washington.

LE GALÈS, P., THATCHER, M. (1995), Les Réseaux de politique publique? Débat autour des "policy networks ", Paris, L'Harmattan.

LEVY, J. D., (2001), "Partisan Politics and Welfare Adjustment: The Case of France ", Journal of European Public Policy, 8 (2), p. 265-285.

LIJPHARDT, A. (1999), Patterns of Democracy. Government Forms and Performance in Thirty-Six Democracies, New Haven (Conn.), Yale University Press.

LINDBLOM, C. E. (1959), "The Science of "Muddling Through" ", Public Administration Review, 19 (2), p. 79-88.

MAIR, P. (2008), "The Challenge to Party Government ", West European Politics, 31 (1-2), p. 211-234.

MANIN, B. (1995), Principes du gouvernement représentatif, Paris, Flammarion.

MARTIN, P. (2010), "L'immigration, un piège pour la droite? ", Commentaires (132), p. 1027-1036.

MEGUID, B. M. (2008), Party Competition Between Unequals: Strategies and Electoral Fortunes in Western Europe, Cambridge, Cambridge University Press.

PARTI SOCIALISTE (1997), "Changeons d'avenir. Nos engagements pour la France. Programme du Parti socialiste pour les élections de 1997 ".

PETROCIK, J. R. (1996), "Issue Ownership in Presidential Elections, with a 1980 Case Study ", American Journal of Political Science, 40 (3), p. 825-850.

PIERSON, P. (1996), «The New Politics of the Welfare State ", World Politics, 48, p. 143-179.

PIERSON, P. (2000), "Path Dependence, Increasing Returns, and the Study of Politics ", American Political Science Review, 94 (2), p. 251-267.

PPC (2010), "L'explication du changement dans les politiques publiques? Identifications, causes et mécanismes ", dans PALIER, B., SUREL, Y. et al. (dir.), Quand les politiques changent. Temporalités et niveaux de l'action publique, Paris, L'Harmattan.

RIKER, W. H. (1986), The Art of Political Manipulation, New Haven (Conn.), Yale university press.

RIKER, W. H. (1993), Agenda formation, Ann Arbor (Mich.), University of Michigan Press.

RIKER, W., CALVERT R. H., MUELLER, J. E., WILSON, R. K. (1996), The Strategy of Rhetoric? 
Campaigning for the American Constitution, New Haven (Conn.), Yale University Press.

ROBERTSON, D. (1976), A Theory of Party Competition, Londres, Wiley.

ROCHEFORT, D. A., COBB, R.W. (1994), The Politics of Problem Definition? Shaping the Policy Agenda, Lawrence (Kan.), University Press of Kansas.

ROSE, R., DAVIES, P. L. (1994), Inheritance in Public Policy: Change Without Choice in Britain, New Haven (Conn.), Yale University Press.

RYU, J. E. (2009), "Exploring the Factors for Budget Stability and Punctuations: A Preliminary Analysis of State Government Sub-Functional Expenditures ", Policy Studies Journal, 37, p. 457-473.

SCHATTSCHNEIDER, E. E. (1960), The Semi-Sovereign People. A Realist's View of Democracy in America, New York (N. Y.), Wadsworth.

SCHMIDT, M. (1996), "When Parties Matter: A Review of the Possibilities and Limits of Partisan Influence on Public Policy ", European Journal of Political Research, 30 (2), p. 155-183.

SCHUMPETER, J. A. (1942), Capitalism, Socialism and Democracy, New York (N. Y.), Harper and Row.

SINÉ, A. (2006), L'Ordre budgétaire ? L'économie politique des dépenses de l'État, Paris, Economica.

STOKES, D. E. (1963), « Spatial Models of Party Competition ", American Political Science Review, 57 (2), p. 368-377.

STROM, K., MÜLLER, W. C. (1999), "Political Parties and Hard Choices ", in MÜLLER, W. C., STROM, K. (eds), Policy, Office, or Votes?, Cambridge, Cambridge University Press, p. 1-35.
SUREL, Y. (1997), L'État et le livre ? Les politiques publiques du livre en France? 1957-1993, Paris, L'Harmattan.

SWANK, D. (1992), "Politics and the Structural Dependence of the State in Democratic Capitalist Nations ", American Political Science Review, 86 (1), p. 38-54.

TARDAN, A. (1988), "Le rôle législatif du Sénat », Pouvoirs, 44, p. 104-110.

TSEBELIS, G. (2002), Veto Players: How Political Institutions Work, Princeton (N. J.), Princeton University Press.

TSEBELIS, G., MONEY, J. (1995), «Bicameral Negotiations: the Navette System in France ", British Journal of Political Science, 25 (1), p. 101-129.

VOLKENS, A. (ed.) (2001), "Manifesto Research since 1979: from reliability to validity ", in LAVER, M., Estimating the Policy Positions of Political Actors, Londres, Routledge, p. 33-49.

WILDAVSKY, A. (1987), "Choosing Preferences by Constructing Institutions: A Cultural Theory of Preference Formation ", American Political Science Review, 81 (1), p. 3-22.

WILDAVSKY, A. (2002), A Comparative Theory of Budgetary Processes. $2^{\ominus}$ éd., Boston (Mass.), Little, Brown.

WILENSKI, H. L. (1975), The Welfare State and Equality. Structural and Ideological Roots of Public Expenditures, Berkeley (Calif.), University of California Press.

ZITTOUN, P. (2001), "Partis politiques et politiques du logement. Échange de ressources entre dons et dettes politiques ", Revue française de science politique, 51 (5), p. 683-706. 